\title{
A comparison of four quality of life instruments in cardiac patients: SF-36, QLI, QLMI, and SEIQoL
}

\author{
H J Smith, R Taylor, A Mitchell
}

\begin{abstract}
Background-With the increasing use of quality of life measures in evaluations of cardiac interventions, criteria are needed for selecting appropriate quality of life measures. An important criterion is the sensitivity of a measure for detecting clinically important changes.

Objectives-To compare the sensitivity of four measures when used in a group of cardiac patients undergoing the same intervention.

Methods-The short form 36 (SF-36), the quality of life index-cardiac version (QLI), the quality of life after myocardial infarction questionnaire (QLMI), and the schedule for the evaluation of individual quality of life (SEIQoL) were used to evaluate quality of life in a group of 22 patients after myocardial infarction or coronary artery bypass graft (CABG), at the beginning of rehabilitation and six weeks later. Analysable data were obtained from 16 patients.

Results-A significant improvement over time was only observed for the SF-36 subscale, vitality $(p<0.05)$. Five of the eight SF-36 subscales and one of the four QLMI subscales showed modest sensitivity (index: $>0.2$ and $<0.5$ ), while all other subscales showed poor sensitivity (index: $<0.2)$. Using SEIQoL, family was most often nominated as an area of importance to quality of life $(n=13)$, followed by health $(n=10)$, leisure/hobbies $(n=8)$, marriage $(n=8)$, and work $(n=6)$.

Conclusions-All four QOL measures used in this study were found to lack sensitivity to change. Further research is needed using other cardiac populations and interventions in order to verify these findings, with a view to developing more sensitive quality of life scales.
\end{abstract}

(Heart 2000;84:390-394)

Keywords: quality of life; heart disease; short form 36; quality of life index-cardiac version; quality of life after myocardial infarction questionnaire; schedule for the evaluation of individual quality of life

Department of Psychology, University of Exeter, Exeter, Devon, UK H J Smith

A Mitchell

Health Services Research Unit, London School of Hygiene and Tropical Medicine, Keppel Street, London WC1E 7HT, UK $\mathrm{R}$ Taylor

Correspondence to: Dr Taylor

rod.taylor@nice.nhs.uk

Accepted 17 June 2000
In the field of cardiology, there has been a long tradition of assessing the effectiveness of new and emerging health technologies. These evaluations have invariably focused on outcome measures such as mortality, morbidity, and clinical function. However, in recent years there has been an increasing use of more patient focused outcomes, in particular quality of life. ${ }^{1}$ Indeed a state of the art review identified some 150 different quality of life measures. $^{2}$

Quality of life measures may be disease (or condition) specific or generic. ${ }^{3}$ Disease specific measures focus on the complaints that are attributable to a specific diagnosis or patient population. In contrast, generic quality of life measures are intended to be broadly applicable across different interventions, and across patients with different characteristics. Another more recent approach to quality of life assessment is the development of so called "individualised" (or patient generated) measures. These measures allow patients, from their own perspective, to identify the aspects that contribute most to their overall quality of life. ${ }^{4}$

With the proliferation of different types of measures and growth in the theoretical literature, criteria are needed to assist clinicians in assessing the suitability of a particular quality of life measure. ${ }^{1}$ Of these published criteria, sensitivity (or responsiveness) - which is the ability of a measure to detect a clinically important change-is probably one of the most important when selecting a measure for a cardiac trial. The choice of a quality of life measure with inadequate sensitivity can result in a false negative trial in which the intervention improves how patients feel, and yet the measure fails to detect the improvement.

Several studies have reported a wide range of sensitivity levels (from very poor to very good) when applying quality of life measures to cardiac populations. $^{5-9}$ Although these studies have generally used similar quality of life measures, it is difficult to compare their results as they have been applied to different cardiac populations and across a variety of medical and surgical interventions. In an attempt to overcome the difficulty of comparing different quality of life measures across different studies, in the present study we proposed to apply a range of quality of life measures to the same group of cardiac patients who were all undergoing the same intervention, in this case an outpatient cardiac rehabilitation programme. We aimed to compare the sensitivity of four differing and widely cited quality of life measures: a generic measure, the short form 36 $(\mathrm{SF}-36)^{10}$; two disease specific measures, the MacNew quality of life after myocardial infarction questionnaire (QLMI), ${ }^{7}$ and the Ferrans and Power quality of life index-cardiac version $\mathrm{III}^{11}$; and a patient generated measure, the 
schedule for the evaluation of individual quality of life (SEIQoL). ${ }^{412}$

\section{Methods}

PATIENTS

Consecutive patients referred to the Royal Devon and Exeter Health Care Trust for cardiac rehabilitation between January and April 1998 were contacted by telephone or letter. This included patients who had had either a myocardial infarct or coronary artery bypass graft surgery (CABG), or both. To be eligible for rehabilitation, patients also needed to be free of medical contraindications, for example chronic chest conditions or severe arthritis.

Twenty two of 25 patients who were invited to take part in the study agreed to participate (88\% response) and gave their informed consent. Of those contacted, only three patients were unable or unwilling to take part in the rehabilitation programme. Three participants discontinued the rehabilitation after one session and were not included in the study. Two participants failed to complete their questionnaires and one was lost to follow up. Therefore the final response rate was $64 \%$, comprising 16 participants (15 men and one women) with a mean age of 61 years (range 43-73 years).

\section{STUDY DESIGN}

The four quality of life measures were administered to participants before they started cardiac rehabilitation (baseline, t1) and six weeks afterwards (follow up, t2). They consisted of three self administered questionnaires which participants completed at home and returned by post, and an interview based measure which took place in a quiet room, following the patients' initial clinical assessment for rehabilitation. At follow up, the interview was adapted and administered by post for most participants.

Information about the participants' diagnosis and the time since the cardiac event was obtained from medical records. Participants were asked to provide a list of the drugs they were taking at baseline and whether they smoked. Additional demographic information on marital status, work status, and education level was also obtained from participants at baseline.

\section{INTERVENTION}

The rehabilitation programme was a comprehensive hospital based cardiac rehabilitation undertaken by a multidisciplinary health professional team that included physiotherapists, occupational therapists, cardiac nurses, and physicians. Participants were encouraged to attend hospital based group sessions twice a week, with each session lasting from two to three hours, over a six to eight week period. During sessions, patients undertook supervised aerobic exercise in the form of circuit training during which they were encouraged to work at an intensity of $60-80 \%$ of heart rate reserve over a 20-40 minute period. Relaxation therapy sessions (20-30 minutes) were undertaken on completion of the exercise, and finally a health care professional led an educational session. Education topics included risk factor modification, the actions and appropriate use of cardiac drugs, and occupational advice. Partners were also encouraged to attend and participate. In addition, patients were additionally requested to undertake one to two further unsupervised aerobic sessions a week. Over the course of six weeks, 10 participants in the study attended at least 10 sessions, while six attended between four and seven sessions. All 16 participants were included in the analyses.

\section{MEASURES}

Short form 36

The SF-36 is a self administered generic measure of health status containing 36 questions. Scores are transformed to a scale of $0-100$, where higher scores represent higher functioning. Using content analysis, the items were assigned to eight subscales: physical functioning, role-functioning, bodily pain, general health, vitality, social functioning, roleemotional, and mental health. ${ }^{10}$

\section{Quality of life index-cardiac version}

The QLI is a self administered disease specific measure, originally developed to measure quality of life in both healthy and ill individuals. ${ }^{11}$ The content validity of the QLI was established by an extensive review of published reports on issues related to quality of life and on reports from cardiac patients regarding the quality of their lives. ${ }^{11}$ The scale has 36 items and four subscales: health and functioning, social and economic aspects, psychological and spiritual status, family and relationships. Two questions from QLI (illustrating its disease specific nature) are: "How important to you is being completely free from chest pain?" and "How important to you is being able to breathe without shortness of breath?" The same 36 items are used to measure levels of satisfaction (part 1) and importance (part 2), and scores from parts 1 and 2 are combined so that higher scores represent higher satisfaction and importance. Scores for each subscale are transformed to a scale of $0-30$.

\section{Quality of life after myocardial infarction}

The QLMI was developed and tested to evaluate a comprehensive cardiac rehabilitation programme for patients after myocardial infarction. ${ }^{7}$ It comprises 26 items that were selected after being rated as important by patients and clinicians. Two questions from QLMI (illustrating its disease specific nature) are: "In the past two weeks, how much time did you feel very confident that could deal with your heart problem?" and "During the last two weeks how much have you been limited in doing sports or exercise as a result of your heart problem?" These represent three subscalesemotional, physical, and social-with scores ranging from $0-7$, where higher scores represent higher functioning.

The above three measures have undergone extensive testing and have shown validity, with high inter- and intrareliability coefficients. ${ }^{12-14}$ 
Schedule for the evaluation of individual quality of life

The SEIQoL was developed as an interview based quality of life measure which allows individuals to nominate, weigh, and assess domains of greatest relevance to their quality of life. ${ }^{12}$ Respondents are required to have sufficient insight into the factors that determine their quality of life, ability to think abstractly, and the ability to make judgements based on information presented in diagrammatic form. The SEIQoL interview begins with a brief talk about the concept of "quality of life" and how it consists of areas in life that have special importance to individuals. The interview then involves three stages:

(1) Cue elicitation. The individual is asked, "What are the five most important aspects of your life at the moment?" If respondents have difficulty thinking of five areas, they are given examples from a list of prompts: "For example, people often mention family, health, work, and leisure activities." The individual's cues are then written down, together with a brief description of what each cue refers to.

(2) Determining the current status on each cue. The individual is presented with a vertical visual analogue scale, ranging from worst possible (0) to best possible (100). They are then asked, "'How would you rate yourself on each of these areas of life at the moment, on a scale from worst possible to best possible?" These ratings are recorded in the form of a bar chart drawn by the interviewee.

(3) Quantification of the relative weighting of each cue. The individual is asked, "How do the five areas compare in importance to each other?" To do this, the interviewee needs to divide $100 \%$ between the five cues and apportion higher weights (\%) to areas of life considered to be more important.

In this study, participants were asked to re-evaluate their original cues at follow up, by following the above procedure, provided in a written format, and administered by post to most participants. Some participants preferred to follow verbal instructions and therefore chose to have the SEIQoL administered once again by an interview at follow up. The SEIQoL is primarily an individual measure, but scores can be combined to produce a global index ranging from $0-100$, where higher scores represent higher quality of life.

STATISTICAL ANALYSIS AND SAMPLE SIZE

Based on the results of a previous study (standardised difference of 1.16$),{ }^{15}$ it was estimated that at least eight participants should be recruited to detect a similar magnitude of difference using a two tailed paired test at 5\% significance and $80 \%$ power. ${ }^{16}$ To assess the sensitivity of the scales for detecting changes in quality of life, a sensitivity index was calculated by dividing the mean change in scores, between baseline and follow up, with their standard deviation at baseline. ${ }^{17}$ Scores greater than 0.2 , 0.5 , and 0.8 were used to represent modest, moderate, and good sensitivity, respectively. ${ }^{17}$
Owing to the non-normal distribution of the subscale scores, the results are reported as medians (and interquartile ranges) and inferential testing was undertaken using nonparametric methods. A case example and a list of the life areas arising from the SEIQoL were drawn up as a means of providing a more qualitative impression of quality of life.

\section{Results}

The baseline clinical and sociodemographic characteristics of the 16 participants are presented in table 1 .

CHANGES IN QUALITY OF LIFE SCORES AND SENSITIVITY

Table 2 shows the median values of the SF-36, QLI, QLMI, and the SEIQoL scales at baseline and follow up. There appears to have been a small improvement in many of the subscale scores over the period of rehabilitation. However, a significant improvement $(p<0.05)$ was only observed for the SF-36 subscale, vitality. None of the subscales achieved moderate sensitivity (index: $>0.5$ ) as measured by the sensitivity index, and only five SF-36 subscales and one QLMI subscale achieved modest sensitivity (index: > 0.2). All other subscales showed poor sensitivity (index: $<0.2$ ).

\section{SEIQOL RESULTS}

Table 3 shows the types of cue nominated by individuals, together with the frequency and percentage of times each cue was nominated. Family was the most frequently nominated cue, followed by health, leisure/hobbies, marriage, and work. The cues represent a wide range of life areas and activities, and no single cue, such as health, was nominated by all participants.

A profile of ratings and weights at baseline and follow up for one individual is shown in fig 1. This individual rated marriage and family as functioning very well, and his assessment

Table 1 Clinical and sociodemographic characteristics of the patients $(n=16)$ at baseline (T1)

\begin{tabular}{|c|c|}
\hline \multicolumn{2}{|l|}{ Variable } \\
\hline Age (years) ${ }^{\star}$ & $61(7.6)$ \\
\hline Time since event (weeks) ${ }^{\star}$ & $20(9)$ \\
\hline \multicolumn{2}{|l|}{ Diagnosis } \\
\hline CABG & 14 \\
\hline Myocardial infarction & 2 \\
\hline Male & 15 \\
\hline Female & 1 \\
\hline \multicolumn{2}{|l|}{ Education } \\
\hline Secondary & 9 \\
\hline Further+ & 7 \\
\hline \multicolumn{2}{|l|}{ Work status } \\
\hline Working & 7 \\
\hline Retired & 9 \\
\hline \multicolumn{2}{|l|}{ Marital status } \\
\hline Married & 16 \\
\hline Single & 0 \\
\hline \multicolumn{2}{|l|}{ Smokers } \\
\hline Yes & 1 \\
\hline No & 15 \\
\hline \multicolumn{2}{|l|}{ Drug treatment } \\
\hline$\beta$ Blocker & 6 \\
\hline ACE inhibitor & 4 \\
\hline Lipid lowering agent & 11 \\
\hline Diuretic & 6 \\
\hline Antiarrhythmic & 2 \\
\hline Vasodilator & 4 \\
\hline Other & 5 \\
\hline
\end{tabular}

Values are ${ }^{\star}$ mean $(\mathrm{SD})$ or $\mathrm{n}$. 
Table 2 Median (upper and lower centiles) for mean baseline ( $t 1)$ and follow up (t2) quality of life (QoL) scale scores with Wilcoxon signed ranks test p values, sensitivity index scores, and ceiling effects

\begin{tabular}{|c|c|c|c|c|c|c|}
\hline & QoL scale & $t 1$ & $t 2$ & $p$ Value & Sensitivity index & Ceiling effect $f$ \\
\hline 1 & SF-36, Role-physical & $25(6$ to 94$)$ & 75 (6 to 100$)$ & 0.08 & 0.4 & $25 \%(4)$ \\
\hline 2 & SF-36, Bodily pain & $57(41$ to 83$)$ & 73 (47 to 96$)$ & 0.11 & 0.28 & $19 \%(3)$ \\
\hline 3 & SF-36, General health & $56(38$ to 83$)$ & $55(48$ to 82$)$ & 0.62 & 0.06 & $6 \%(1)$ \\
\hline 4 & SF-36, Vitality & 55 (36 to 79$)$ & 70 (43 to 84$)$ & $0.04^{\star}$ & 0.27 & Nil \\
\hline 5 & SF-36, Social functioning & $88(66$ to 100$)$ & $100(75$ to 100$)$ & 0.14 & 0.32 & $31 \%(5)$ \\
\hline 6 & SF-36, Role-emotional & $100(67$ to 100$)$ & $100(50$ to 100$)$ & 0.72 & 0.07 & $69 \%(11)$ \\
\hline 7 & SF-36, Mental health & $76(65$ to 92$)$ & $88(66$ to 92$)$ & 0.40 & 0.16 & $13 \%(2)$ \\
\hline 8 & SF-36, Physical functioning & $83(66$ to 89$)$ & $85(71$ to 95$)$ & 0.12 & 0.22 & Nil \\
\hline 9 & QLI, Health and functioning & 21 (19 to 26$)$ & $24(19$ to 27$)$ & 0.63 & 0.03 & $6 \%(1)$ \\
\hline 10 & QLI, Socioeconomic & $24(21$ to 28$)$ & $25(21$ to 29$)$ & 0.37 & 0.01 & $19 \%(3)$ \\
\hline 11 & QLI, Family & 29 (24 to 30$)$ & $29(26$ to 30$)$ & 0.14 & 0.06 & $50 \%(8)$ \\
\hline 12 & QLI, Psycho/spiritual & 24 (18 to 28$)$ & $24(20$ to 29$)$ & 0.12 & 0.14 & $19 \%(2)$ \\
\hline 13 & QLI, Overall & $23(20$ to 27$)$ & $25(20$ to 27$)$ & 0.53 & 0.12 & $6 \%(1)$ \\
\hline 14 & QLMI, Emotional & $5.8(4.8$ to 6.2$)$ & $5.7(4.4$ to 6.6$)$ & 0.92 & 0 & $13 \%(2)$ \\
\hline 15 & QLMI, Physical & $5.6(4.9$ to 6.2$)$ & $5.9(5.0$ to 6.3$)$ & 0.16 & 0.15 & Nil \\
\hline 16 & QLMI, Social & $5.6(5.1$ to 6.1$)$ & $5.8(4.9$ to 6.7$)$ & 0.29 & 0.26 & Nil \\
\hline 17 & QLMI, Total & $5.5(5.0$ to 6.2$)$ & $5.8(4.8$ to 6.5$)$ & 0.36 & 0.13 & Nil \\
\hline 18 & SEIQoL & $82(69$ to 84$)$ & $81(71$ to 94$)$ & 0.84 & 0.10 & $13 \%(2)$ \\
\hline
\end{tabular}

The sensitivity index was calculated as the ratio between the mean change in scores from $\mathrm{t} 1$ to $\mathrm{t} 2$ and the standard deviation of the baseline mean scores. Values $>0.2$ $=$ modest sensitivity; $>0.5=$ moderate sensitivity; $>0.8=$ good sensitivity.

${ }^{\star} \mathrm{p}=0.05$.

†The ceiling effect for each subscale is shown by the $\%$ and number of subjects with a maximum score at baseline.

Table 3 Frequency (percentages) of cues nominated by participants as most important to their overall quality of life

\begin{tabular}{lr}
\hline Elicited cues & Frequency \\
\hline Family & $13(81 \%)$ \\
Health & $10(62 \%)$ \\
Leisure/hobbies & $8(50 \%)$ \\
Marriage & $8(50 \%)$ \\
Work & $6(38 \%)$ \\
Exercise & $4(25 \%)$ \\
Home life/environment & $4(25 \%)$ \\
Social life & $4(25 \%)$ \\
Independence & $4(25 \%)$ \\
Food & $3(19 \%)$ \\
Finances & $3(19 \%)$ \\
Miscellaneous & $3(19 \%)$ \\
Holidays/travel & $2(13 \%)$ \\
Pets & $2(13 \%)$ \\
Work related activity since retirement & $2(13 \%)$ \\
\hline
\end{tabular}

*Cues nominated by only one participant were labelled as miscellaneous and included dining out, church, and retirement.

\section{Time 1 \\ $\square$ Time 2}

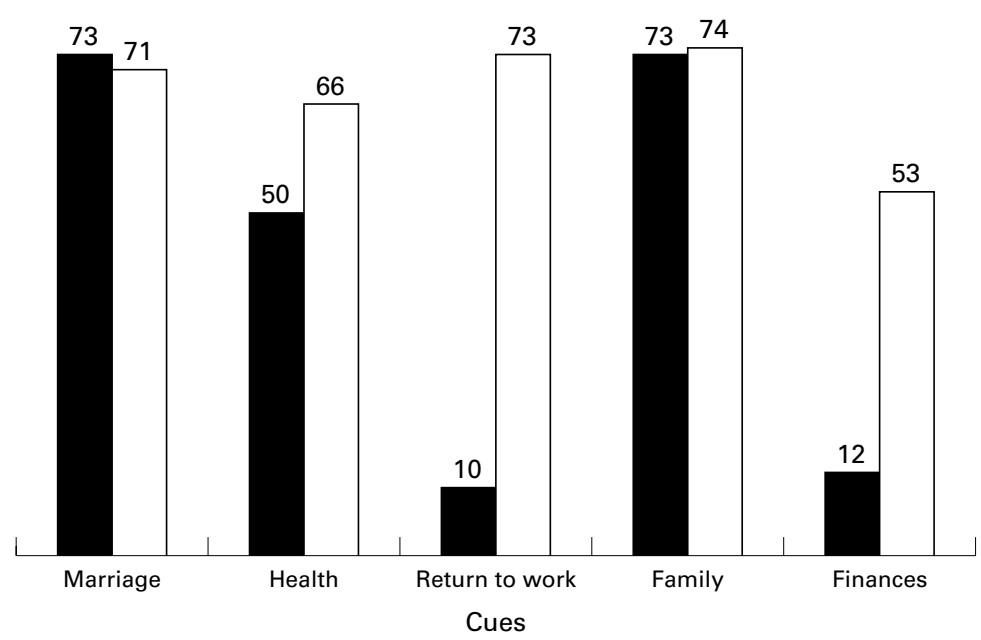

Figure 1 SEIQoL cue levels (on a scale of 0-100, where higher scores reflect higher quality of life) for one patient at time 1 and time 2. Cue weights at times 1 and 2: marriage $35 \%$; health $30 \%$; return to work $10 \%$; family $20 \%$; finances $5 \%$. remained stable at follow up. Health was perceived to have improved at follow up, and considerable improvements were also perceived for returning to work and finances. Marriage was regarded as the most important cue, followed by health, family, and return to work, while finances were regarded as the least important.

\section{Discussion}

The purpose of this study was to compare the sensitivity of four commonly used quality of life measures in a group cardiac patients who were all undergoing cardiac rehabilitation. The results showed generally good agreement across the subscales of the four quality of life instruments, but they had only poor to modest levels of sensitivity. It has been suggested that disease specific measures have superior levels of sensitivity than their generic counterparts. ${ }^{35}$ Although all measures performed poorly, surprisingly the two disease specific measures in this study (QLI and QLMI) had the lowest levels of sensitivity.

There are two potential limitations in the design of this study that may explain the finding of lack of sensitivity. First, it may be argued that the number of patients assessed was insufficient to show a change during the period of study. However, we propose that this is unlikely, given that the study sample size was based on a formal power calculation from the results of a previous study evaluating change in quality of life using a similar population of cardiac patients and a similar intervention. ${ }^{15} \mathrm{Sec}-$ ond, it is possible that the particular intervention evaluated in this study (a six week outpatient cardiac rehabilitation programme) was inadequate to bring about improvements in patient's quality of life. Again, this seems to have been unlikely, given that previous studies have shown improvements in quality of life with rehabilitation programmes of similar content and duration..$^{515}$ It is important to note that different quality of life instruments or methods (for example, the Nottingham health profile and the time trade off technique) were used in these two previous studies. 
We propose that the principal reasons for the lack of sensitivity of the quality of life measures in this study are both the characteristics of the population studied and the inherent limitations in the measures themselves. It has been suggested that the inability of quality of life measures to show change can be explained by a so called ceiling effect, in which the quality of life subscales indicate the complete absence of any dysfunction and therefore cannot show further improvement. ${ }^{4}$

Although there was some evidence of such a ceiling effect in this study (more than one third of the patients with a maximum score), this effect was limited to only three of the 18 subscales (SF-36 social functioning, SF-36 role-emotional, and QLI family). However, on many of the subscales, the scores were at the higher end of the scales. This is illustrated by the four QLMI subscales, all of which had a median score in excess of 5.5 (out of seven) at baseline, despite having a low proportion of patients with a maximum score. This is also evident by the higher SF-36, QLMI, and QLI scores in the patients in this study compared with those reported at baseline in other studies of rehabilitation ${ }^{15}$ or antianginal interventions. ${ }^{9}$

Turning attention to the instruments themselves, it is interesting to note that in the case of the patient generated interview measure (SEIQoL), patients nominated family as an important quality of life domain more often than health. Of the other quality of life measures, the only one with a subscale that comprehensively covers family issues was the disease specific QLI. This subscale appeared to measure a separate domain, as it showed no association with any of the other subscales.

The SF-36 role-physical subscale produced the highest (though still modest) sensitivity score. However, caution is needed when interpreting this, as the questions on this subscale are dichotomous, requiring a yes or no answer, and only ask participants if they have had any problems with activities as a result of their physical health. Even with a dramatic improvement in scores, the amount of improvement cannot be quantified by such questions. The QLI and QLMI avoid this problem by using a Likert scale which enables respondents to rate their degree of satisfaction or the presence of a disease specific problem along a scale ranging from 1 (very unimportant) to 6 (very important) for the QLI, and from 1 (none of the time) to 6 (all of the time) for the QLMI. Another problem when comparing the sensitivity of the SF-36 with other measures is that its subscales "bodily pain" and "social functioning" comprise only two questions each. Therefore any change in answers from baseline to follow up are more likely to alter scores significantly than a subscale containing more items.

In conclusion, this study showed a lack of sensitivity in all four quality of life instruments. This finding has important implications to the assessment of quality of life in the future assessments of cardiac interventions. There is an urgent need for researchers and clinicians to develop more sensitive quality of life measures for use with cardiac populations. In the meantime, we would recommend caution in the selection, application, and interpretation of quality of life measures used in effectiveness studies involving cardiac patients.

1 Guyatt GH, Naylor DC, Juniper E, et al. User's guides to the medical literature XII How to use articles about health-related quality of life. $\mathcal{F} A M A 1997 ; 277: 1232-7$.

2 Gill TM, Feinstein AR. A critical appraisal of the quality of quality of life measures. $\mathcal{F} A M A$ 1994;272:619-26.

3 Patrick DL, Deyo RA. Generic and disease-specific measures in assessing health status and quality of life. Med Care 1989;27:S217-32.

4 Hickey AM, Bury G, O'Boyle CA, et al. A new short form individual quality of life measure (SEIQoL-DW): application in a cohort of individuals with HIV/AIDS. BMF 1996; 313:29-33.

5 Taylor R, Kirby B, Burdon D, et al. The assessment of recovery in patients after myocardial infarction using three generic quality of life measures. $\mathcal{F}$ Cardiopulmonary Rehabil 1998;18:139-44

6 Dempster M, Bradley J, Wallace E, et al. Measuring quality of life in cardiac rehabilitation: comparing the Short Form 36 and the Nottingham Health Profile. Coronary Health Care 1997;1:211-17.

7 Hillers TK, Guyatt GH, Oldridge N, et al. Quality of life after myocardial infarction. F Clin Epidemiol 1994;47:128796.

8 Spertus JA, Winder JA, Dewhurst TA, et al. Monitoring the quality of life in patients with coronary artery disease. $A m \mathcal{F}$ Cardiol $1994 ; 74: 1240-44$.

9 Dougherty CM, Dewhurst T, Nichol P, et al. Comparison of three quality of life instruments in stable angina pectoris: Seattle angina questionnaire, short form health survey (SF-36), and quality of life index-cardiac version III. $\mathcal{F}$ Clin Epidemiol 1998;51:569-75.

10 Ware JE. SF 36: a valid, reliable assessment from the patients' point of view. $B M F$ 1 1993;306:1429-86.

11 Ferrans C, Powers, M. Quality of life index: development and psychometric properties. Adv Nurs Sci 1985;8:15-24

2 O'Boyle A, Browne J, Hickey A, et al. Administration manual. Dublin: Department of Psychology, Royal College of Surgeons of Ireland, 1995.

13 McHorney CA, Ware JE, Lu JFR, et al. The MOS 36-item short-form health survey (SF-36). III. Tests of data quality, scaling assumptions, and reliability across diverse patient groups. Med Care 1994;32:40-66.

14 Ferrans C, Powers M. Psychometric assessment of the quality of life index. Res Nurs Health 1992;15:111-19.

15 Oldridge N, Gottlieb M, Guyatt G, et al. Predictors of health-related quality of life with cardiac rehabilitation after health-related quality of life with cardiac rehabilitation after 1998; 18:95-103.

16 Machin D, Campbell M, Fayers P, et al. Sample size tables for clinical studies, 2nd ed. Oxford: Blackwell Science, 1997: $24-5$

17 Kazis L, Anderson J, Meenan R. Effect sizes for interpreting changes in health status. Med Care 1989;27(suppl 3):S17889.

18 Jolliffee J, Taylor RS, Oldridge N, et al. The effectiveness of exercise-based rehabilitation for cardiac patients [protocol]. Oxford: The Cochrane Library [on line]: http:// www.updateuse.com/clip/clib.htm 